\title{
Research on Risk Control Management Process in the Cloud Computing Environment
}

\author{
Li Ma \\ School of Finance, Guizhou University of Finance and Economics, Guiyang Guizhou, \\ 550025, China
}

\begin{abstract}
With the rapid development of information and communication technology (ICT) over the past half century, the cloud computing model is one of the most controversial issues in the field of ICT. In addition, cloud computing plays a vital role in today's business world, but no risk management process is incorporated into the cloud computing innovation technology, so in this paper, a cloud management environment in the implementation of the risk management process Conceptual model. It highlights the different processes of risk management (risk identification, risk analysis, risk planning, risk enforcement and risk monitoring), and how their impact affects the cloud environment to ensure proper protection of data and information as well as cloud and connectivity reliable.
\end{abstract}

\section{Keywords}

Cloud Computing; Cloud Computing Risks; Information and Communications Technology (ICT); Risk Management

\section{云计算环境中运行风险管理流程研究}

\author{
马莉 \\ 贵州财经大学金融学院, 贵阳 50025, 中国
}

摘要：随着信息通信技术（ICT）在过去半个世纪的迅速发展，云计算模式是目前 ICT 领域 最受争议的议题之一。此外, 云计算在当今的商业世界中起着至关重要的作用, 然而没有 风险管理流程结合到云计算的创新技术中，因此，在本文中提出了一个在云计算环境中实施 风险管理流程的概念模型。它突出强调了风险管理（风险识别, 风险分析, 风险规划, 风险 执行和风险监控）的不同过程，以及它们的存在如何影响云环境，以确保数据和信息的正确 保护以及云网络和连接是安全可靠的。

关键词：云计算；云计算风险；信息通信技术（ICT）；险管理

云计算通过互联网使用远程服务

1. 引言 器来提供按需访问信息设施。此外, 云计算已成为大数据应用中越来越受 
欢迎的手段，但云计算服务模式和云 计算部署模式中的风险管理流程问题 确实缺乏学术上的研究。本文尝试通 过解决云计算相关的问题, 并提出了 将风险管理流程作为云计算服务模型 和云计算部署模型中有效模型的可靠 技术。本文的研究重点是云计算风险 管理, 由于几乎没有研究论文表明风 险管理过程对云计算服务模型和云计 算部署模式的影响。因此, 本文的目 的是阐明建立概念模型, 并讨论云计 算领域当前的趋势。随着现代社会的 进步，云计算实践正在快速发展，通 常是企业应对不断变化的需求的有效 途径。然而, 对于云供应商的风险管 理实践缺乏信心, 导致了一些企业面 对云计算时的犹豫。尽管存在威胁, 大多数企业或用户也没有计划如何处 理这些威胁。因此需要采用可用于处 理这些威胁的风险管理流程工具, 能 成功实施云计算需要对新出现的风险 进行正确的规划和了解。

\section{2. 云计算}

\section{1. 云计算概念}

按照国家标准的定义, 云计算的 概念是允许合适的、按需的模型网 络, 访问可共享的可配置计算资源池

(如: 网络, 服务器, 存储, 应用和 服务)。同样, 对传播学而言, 云计 算也是一种通过互联网按付费使用可 扩展 IT 服务的新方法。而 Rashmi等

（2013）[1]建议云计算的定义应该是基 于两个观点: 第一, 从用户的角度来 看, 即云计算获得的计算服务, 完全 不需要深入了解使用了哪些底层技 术。其次, 从组织的角度来看, 云计 算提供服务以客户和业务要求为基本 方式, 提供无限制的、差异化的服务 质量, 以促进快速创新和决策。而赞 助组织委员会 $(\mathrm{COSO})$ 使用的概念性
的定义即云计算是一种计算资源（网 络, 服务器, 存储, 应用和服务) 用 于实现个别组织或团体通过互联网连 接从任何位置使用和获得其计算资源 和不同应用。

\section{2. 云计算的优势}

云计算的优点包括改善了通过互 联网服务的传递, 具有成本效益, 加 速了创新, 缩短了上市时间。另外, 云计算包含了重要的敏感数据, 如个 人, 政府或企业数据等。因此, 云计 算系统必须比传统制度得到更加严密 的保护。此外, Karadsheh和 Alhawari （2011）人为云计算对于各种规模的 业务来说都是重要课题 ${ }^{[2]}$ 。云计算具有 可扩展性, 安全性, 低成本, 便携性 和可用性等优点。

除了与之相关的优势, Ismail （2011）从服务角度来总结了云计算 的五个特点 ${ }^{[3]}$ : 首先, 云计算是基于互 联网提供的服务; 其次, 云计算受到用 户的信赖, 可随时访问和联系数据; 第三, 它使用户能够在使用数据的任 何时候都受到法律控制; 第四, 它提 供源了虚拟化; 最后, 云计算使用户 利用服务遵守合同协议。

文中列举了许多云计算的相关优 点。最近, Fan等人 (2012) 指出 ${ }^{[4]}$, 云计算的概念已经彻底改变计算机系 统的架构。因此, 主要的优点是机构 和组织可以降低成本, 节省能源, 并 通过更换具有云计算服务的设施来自 动升级传统的计算机系统。除了这些 优势, 云计算还可以在更加可靠, 扩 展性和可用性更强的分布式环境下, 通过互联网传递按需的计算服务。

\section{3. 云计算服务模型}

云计算服务的分类为读者更好地 理解本文提供了基础。根据NIST、 Mell和Grance（2011）对云计算提出的 分类方法 ${ }^{[5]}$, 提供商向云计算客户提供 
三种不同类型的服务。首先, 软件即 服务（SaaS）; 其次, 平台即服务 （ PaaS）; 最后是基础架构即服务 (IaaS的。主要包括亚马逊, 谷歌, 销 售部门, 雅虎和微软等云计算提供 商。图1显示了云计算的三个分类服务 模式。它显示了若干风险管理流程的 责任与三种服务模式有关。

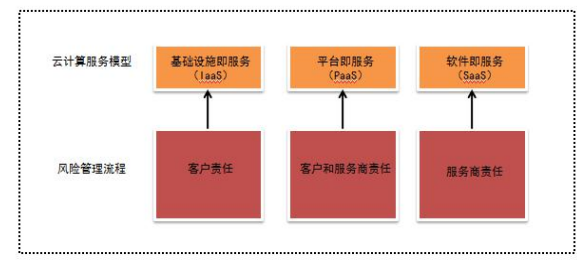

图1 云计算服务模型的风险管理流程

软件即服务（ $\mathrm{SaaS}$ ）：指基于用 户的云计算层利用网络汶览器访问其 他人开发, 维护和提供的软件服务。 此外, 客户不具有管理并控制云应用 的基本能力。平台即服务（PaaS）: 指提供计算中间件的提供商。客户使 用一系列由 Paas 提供商支持的编程语 言和工来改善他们自己的软件和应用 程序。另外, 客户可以自由构建自己 的应用程序。基础架构即服务

（IaaS）：指供应商允许其消费者达到 不同类型的基础设施, 如存储, 内存 和 CPU 处理能力, 并通过使用自动扩 展的虚拟机来安排和运行应用程序。 此外, 客户能够组织和运行任意软 件, 其中可以包括操作系统和不同的 应用程序。

\section{4. 云部署模型}

在本节中, 我们介绍了云部署模 型的分类。而云服务部署的主要方式 有四种, 即私人, 公共, 社区和混合 云:

（1）私有云，这种云服务模式特 别适用于一个组织并由第三方或组织 经营。这些服务可能存在于非现场。 另外, 私有云的结构由组织和第三方
提供商维护, 其基础设施可以位于外 部和内部。

（2）公共云，这种云服务模式是 公众可以访问的, 并且是由拥有销售 云服务的组织或机构, 如 Amazon Cloud。这种类型通常是由第三方提供 商管理。公共云的结构也由第三方供 应商拥有。公共云的这种基础设施位 于外部和内部。

（3）社区云，这种云服务模式常 见于某个组织协助一个共同关心的特 定社区, 如安全要求、政策和使命。 社区云的一个重要示例包括 Facebook。

（4）混合云，这种云服务模式是 不同云组成的计算基础设施（公共或 私人社区）, 由组织和组织管理第三 方提供商。结构位于外部位置。其中 每一个都需要风险管理流程, 以及由 第三方提供商管理的风险（见图 2）。

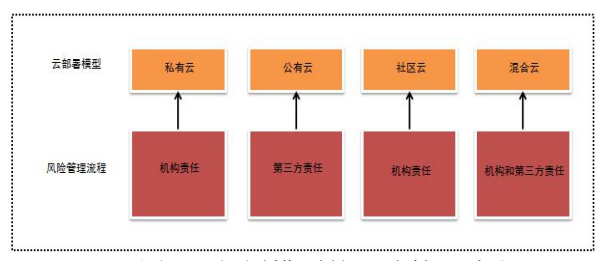

图2 云部署模型的风险管理流程

据国际数据公司的调查显示, 亚 太地区 $53 \%$ 的组织正在使用一些云计 算服务模式，其余 $47 \%$ 的组织在未来 12 个月内有计划实施私有或公共云服 务。因此, 一旦组织决定移动 ICT 服务 云, 他们就应该对他们的数据开始分 类, 并为每个云部署模型确定最好的 风险管理过程。

\section{5. 风险概念}

企业将风险管理视为在各种 IT 服 务中控制和管理风险的必要条件。因 此, 风险管理的目的是确定所有风 险, 并制定一个综合计划, 将风险降 至可接受水平。最近, Desai（2015） 
认为 ${ }^{[6]}$, 通用风险可以定义为可能会产 生与预期有偏差的不确定因素的风 险。此外, 风险是由于威胁或可能对 操作产生重大影响的脆弱性而导致出 现问题的可能性。

\section{6. 风险管理流程模型}

每个过程中的风险管理可以为其 成功实施提供更大的可能性。最近,

Lee（2014）将风险管理过程描述为风 险的识别[7], 评估和优先级, 然后协调 和经济地应用资源来减少，监测和控 制不幸事件的可能影响或最大限度地 实现机会。

此外，根据项目管理研究所描述 风险管理流程分为以下几个方面: 1) 风险规划: 决定企业的风险管理活动 方式, 计划和执行情况;2) 风险识 别: 确定哪些风险可能影响业务并确 定其特征；３）定性风险分析：通过评 估和组合其发生和效应的可能性来优 先考虑后续进一步分析的风险;4）定 量风险分析：统计分析对确定风险的 总体业务目标的影响; 5) 风险响应规 划: 制定选择和行动, 以提高机会并 降低业务目标的风险；6）风险监测与 控制: 跟踪已确定的风险, 监测剩余 风险, 识别新风险, 执行风险应对计 划并评估其整个业务的效率。

\section{7. 云计算风险}

云计算包含诸如个人, 政府或商 业数据等重要数据, 因此吸引着黑客 的关注。最近, Albakri 等人的一项研

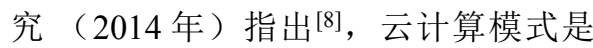
将不同的计算资源作为实用程序分 配, 就像电力一样, 终端用户无需担 心如何管理这些资源。因此, 一般的 研究工作。。是为了描述云计算风 险。从这一点来看, 在服务, 数据和 基础设施层面上, 必须考虑云计算环 境中的风险。
此外, 云计算包括任何考虑此领 域安排的组织进行评估和管理重要的 风险。风险管理主要集中在云计算的 主要风险领域, 如信息安全, 特权用 户访问, 监管合规性和数据位置, 探 索性支持, 可访问性和灾难恢复以及 提供商锁定和长期可行性。在另一项 研究中, 安全风险分为七个部分: 特 权用户访问, 监管, 合规性, 数据位 置, 数据隔离, 恢复, 调查支持和长 期可行性。

而 Tot 等 (2015) 将风险管理描 述为云计算的良好做法 ${ }^{[9]}$ 。主要内容包 括识别、定位, 评估和安全风险的缓 解, 通过选择和实施云计算领域的最 佳实践安全控制。此外可以使其潜在 的快速扩展性, 远程数据存储和使用 共享服务的风险永久化, 这些都有助 于数据保护。

在风险对策中, 优素福和阿莱格 尔 (2012) 将云计算风险及其对策分 为六类 ${ }^{[3]}$ : 一是特权用户访问, 监控授 权用户行为的对策, 限制管理员聘用; 其次, 数据位置, 通过向客户提供有 关其信息存储和处理位置的信息的对 策; 三是数据隔离, 采用加密对策防止 数据大小; 第四, 数据残差表示, 使用 云服务后确保数据删除的对策; 五是数 据恢复, 其他数据中心备份数据对策; 最后, 长期可用, 不再提供云服务时 应用保险的对策。

\section{3. 研究模型}

与先前的研究结合在一起, 作者 提出将概念风险管理纳入云计算环境 模型中, 以描述云计算如何成功。另 外, 根据国家标准与技术研究所的统 计, 有四种不同云计算部署模式, 即 私有, 社区, 公共和混合。还有在特 定云计算中使用的三种不同的云计算 服务模型部署模式。这些云计算服务 模式是 $\mathrm{SaaS}$ (软件即服务) , PaaS 
(平台即服务) 和 $\mathrm{IaaS}$ (基础架构即 服务）。云计算环境中的主要挑战是 有效地解决与四个云计算部署模型和 三个云计算服务模型相关的风险管理 流程问题。

因此, 本文提出并开发了一个概 念性风险管理流程, 以进入云计算环 境模型, 如图 3 所示。此外, 为了将 概念风险管理流程整合到云计算环境 中, 系统支持利用该模型。本节将讨 论各个方面。

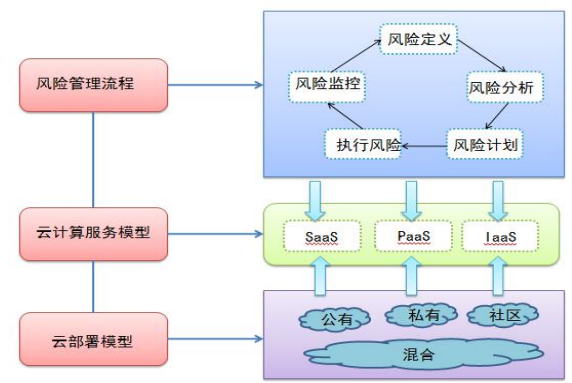

图 3 云计算环境下的风险管理模型

云计算环境中的风险管理在云计 算服务模型和云计算部署模式中经常 被忽略, 但它可以通过提供风险管理 流程 (风险识别, 风险分析, 风险规 划, 风险执行) 中的选择来帮助改善 云计算的成功和风险监控。

云计算环境中的风险识别：在这 个阶段, 我们需要确定哪些风险可能 对云计算环境产生影响, 并记录每个 风险的特征。在这个阶段, 我们还需 要确定和确定每个云计算服务的风险 管理流程的责任。首先: 基础设施即 服务（IaaS），风险识别的责任可能与 客户有关。其次: 软件即服务

（SaaS），责任可能与供应商有关。 最终：平台即服务（PaaS），责任可 能是客户和提供商共同承担。

云计算环境下的风险分析：在这 个阶段, 我们需要进行定性和定量的 风险分析, 以便根据云计算环境中的 可能性和影响对风险进行排序。
云计算环境下的风险规划：在这 个阶段, 我们需要提高在云计算环境 中取得成功的机会, 并减少任何威 胁。我们还需要评估云计算如何与组 织的目标一致。此外, 大多数 SaaS 提 供商都提供应用程序接口（API）调用 读取 (从而导出) 数据记录。此外, PaaS 锁定发生在 API 层（即平台特定 API 调用) 和组件级。最后, IaaS 计算 提供商通常提供基于虚拟机管理程序 的虚拟机 ${ }^{[10]}$ 。

云计算环境中的风险执行: 在此 阶段, 我们需要估计与云计算环境相 关的风险行为, 以确定风险的潜在影 响, 以确保风险规避并实现风险缓 解。

云计算环境中的风险监控: 在此 阶段, 我们需要监控风险, 列出任何 新的潜在风险, 并评估整个云计算环 境中风险方法的效率。此外，关于基 础架构即服务（IaaS），如果云提供商 有服务中断, 此阶段需要监控选项以 尽量减少影响。此外, 平台即服务 ( PaaS) 需要在发生安全漏洞的情况 下监控机密性, 隐私和法律责任（因 为数据库的敏感信息现在将被托管在 场外）。最后，软件即服务 ( SaaS) 需要监控拥有应用程序的人员以及他 们所在的地方。此外, 云计算是一个 高度动态的业务。许多新的业务结构 和挑战可能会在未来几年内发生变 化。因此, 云计算环境中的风险监控 的有效性可以保证在未来可以不断进 行调整和开发。

\section{4. 影响结果}

在当今竞争激烈和快速变化的全 球经济中, 全球经济出现重大动荡。 尽管有云计算环境的优势, 但在机构 兼并、重组等过程中面临的新业务并 不享有这些优势, 因为他们不确定如 何能够在新的业务中建设这些风险的 
影响。因此, 必须采取可用于处理这 些风险的风险管理流程。为了应对云 计算所涉及的许多挑战, 各种业务的 关键解决方案是使用综合风险管理流 程来提供可靠的响应, 以应对与采用 云计算相关的各种风险在一个企业 内。这是一个风险管理流程, 在识别 各种风险方面非常有效, 并提出可帮 助企业应对的适用回应。

此外, 云计算环境是一种景观改 变技术, 其采用率正越来越多。云计 算环境没有进行充分的风险管理流 程, 因此风险管理者需要深入了解云 计算和其核心内容。此外, 该公司必 须决定在云计算环境中引入什么类型 的新业务。基于云计算环境中实施风 险管理流程的提出模式, 公司将决定 哪种服务模式最适合 (即软件即服务

$(\mathrm{SaaS})$ ，平台即服务（PaaS））。 和基础架构即服务（IaaS））。然后, 必须执行风险管理过程, 以确定所选 服务模型可能发生什么类型的风险。 风险管理流程 (风险识别, 风险分 析, 风险规划, 风险执行和风险监 控）必须根据服务类型（ SaaS, PaaS 和 IaaS) 进行量身定制。然后, 公司 需要选择部署模式 (即私有, 公共, 社区和混合云），这些都取决于提出 的新机遇和目标是什么。

\section{5. 结论}

由于云计算被认为是向外部用户 提供不同的服务, 因此需要对提供商 进行补偿以分享其资源和能力。在本 文中提出了一个在云计算环境中实施 风险管理流程 (风险识别, 风险分 析, 风险规划, 风险执行和风险监 控）的概念模型;特别是云计算服务模 式: SaaS (软件即服务) , PaaS (平 台即服务) 和 IaaS (基础架构即服 务）以及云计算部署模式: 私有, 社 区, 公共和混合。另外, 组织也应该
选择一个可以满足其风险管理标准的 云提供商。基于本研究的主题, 本文 总结认为, 将云计算环境中的风险管 理流程实施为处理风险的最广泛分 析。最后, 云计算环境中的风险管理 流程应该能够考虑云服务提供商和云 客户。

\section{参考文献}

[1] 张璐. 中小企业在云计算下如何推 进财务管理信息化研究 $[\mathrm{J} / \mathrm{OL}]$, 中 小企业管理与科技(上旬刊)C. F. , 2017,(10):1-3(2017-10-12).

[2] 陈鹤阳. 基于元分析的云计算用户 采纳行为影响因素研究 $[\mathrm{J} / \mathrm{OL}]$. 图书 馆杂志，2016，11:1(2016-11-18).

[3] 刘艳明. 云计算环境下某银行信息 系统运维项目的风险管理研究 [D]. 中国科学院大学(工程管理与信息 技术学院), 2016.

[4] 兰洁. 云计算对信息安全的影响及 对策分析 $[\mathrm{J} / \mathrm{OL}]$. 电子世界, 2016.

[5] 张秋瑾. 云计算隐私安全风险评估 [D].云南大学, 2015 .

[6] 王美虹. 云计算环境下供应链信息 协同风险控制研究 [D]. 海南大 学, 2015 .

[7] 朱圣才. 基于云计算的信息安全风 险分析与探索 $[\mathrm{J}]$. 西安邮电大学学 报,2013,18(04):89-94.

[8] 周小萌. 基于风险元传递理论的云 计算风险模型研究 $[\mathrm{D}]$. 华北电力大 学, 2013 .

[9] Mouna Jouini,Latifa Ben Arfa Rabai. Comparative Study of Information Security Risk Assessment Models for Cloud Computing systems[J]. Procedia ComputerScience,2016,83:.

[10] Luping Chen,Yongzhen Guo,Feng Zhou. Security Risk Assessment of Mobile Internet Under Cloud Computing Mode[M].Springer Berlin Heidelberg:2014. 